\title{
IN UTERO TRANSPLANTATION OF HUMAN STEM CELLS \\ USING AN ANIMAL MODEL: A COMPARISON BETWEEN \\ TWO TECHNIQUES
}

\author{
Daria GROZA*, PhD Student \\ Emoke PALL**, PhD Student \\ Assistant Mihai CENARIU**, PhD \\ Assistant Raul POP**, PhD \\ Professor Nicolae COSTIN***, PhD \\ Professor loan GROZA**, PhD
}

\begin{abstract}
Objective: The purpose of this study was to assess the feasibility of in utero stem cell transplantation of human umbilical cord blood stem cells in fetal sheep and to compare two different techniques of in utero transplantation, namely ultrasound-guided in utero transplantation and in utero transplantation after midline celiotomy.

Study design: Umbilical cord blood units were collected from term deliveries, after obtaining written informed consent. Human cord blood-derived, CD34+ stem cells were injected into the peritoneal cavity of 60- to 65-day-old ovine fetuses by using 2 different techniques: ultrasound-guided transabdominal percutaneous needle puncture and midline celiotomy with the exposure of the pregnant uterus. Engraftment was determined after birth by flow cytometry with use of human-specific anti-CD 34/45 antibodies.

Results: We obtained a total of 3 chimeric lambs. Using the midline celiotomy technique the fetal loss rate was $75 \%$ and only $33,3 \%$ when using ultrasound-guided transabdominal percutaneous needle puncture technique. Engraftment of donor cells was found in all fetuses, with a mean level of $1.4 \%$ in fetal peripheral blood and $3.3 \%$ in fetal bone marrow.

Conclusion: This preliminary study indicates that in utero stem cell transplantation of human hematopoietic cord blood stem cells in fetal lambs is feasible and effective in terms of hematopoietic engraftment. We also concluded that the ultrasound-guided transabdominal percutaneous needle puncture technique is more effective than performing a midline celiotomy in terms of fetal loss rate.
\end{abstract}

Keywords: stem cells, umbilical cord blood, in utero stem cell transplantation, sheep chimeras

\footnotetext{
* "Iuliu Hațieganu" University of Medicine and Pharmacy, Faculty of General Medicine, Department of Obstetrics and Gynecology "Dominic Stanca”, Emergency Hospital "Prof. Dr. O. Fodor", Cluj-Napoca. 57 Blv. 21 Decembrie Street, 400124 Cluj-Napoca, Romania, e-mail: dariagroza@yahoo.com

** University of Agricultural Sciences and Veterinary Medicine, Faculty of Veterinary Medicine, Department of Veterinary Reproduction, Obstetrics and Gynecology, 3-5 Mănăştur Street, 400372 Cluj-Napoca, Romania

*** "Iuliu Hațieganu" University of Medicine and Pharmacy, Faculty of General Medicine, Chef of Department of Obstetrics and Gynecology "Dominic Stanca", Emergency Hospital "Prof. Dr. O. Fodor", Cluj-Napoca. 


\section{Introduction}

Stem cell transplantation has become a valid treatment option for genetic and malignant diseases of the hematopoietic and immune systems (1). In utero stem cell transplantation is a promising potential therapy for a number of these disorders and has several potential advantages over postnatal treatment (2). Its advantages are based on the unique opportunity provided by the normal haematological ontogeny. The early fetus is immunological immature and thus would theoretically accept foreign antigens (3). Additionally, once successfully transplanted, the intrauterine environment would protect the fetus during ongoing gestation from surrounding viral and bacterial infections. The major advantage would result from the early transplantation before definitive organ damage has occurred $(3,4)$.

The development of an animal model of in utero transplantation of human stem cells has allowed investigators to study hematopoietic differentiation of human progenitor and stem cells (5). In addition, this system provides the opportunity to develop in utero stem cell transplantation as a therapy for genetic disorders of the human fetus (6).

The fetal sheep model has the advantages of similarity in development of fetal immunocompetence relative to gestational age compared with the early human fetus. Though the development of the immune system is not identical, the fetal lamb is tolerant to xenogeneic (eg human) stem cell transplantations before day 70 of gestation $(7,8)$. Furthermore, the relatively long gestation allows the study of different strategies and repetitive transplantations (5).

In contrast to the small animal models, sheep have the advantage of body size. This allows studying technical issues, such as the ultrasound-guided collection of stem cells from the early ovine fetus or the injection techniques $(9,10)$.

There are several routes and techniques of performing in utero stem cell transplantation: ultrasoundguided transabdominal percutaneous needle puncture of the peritoneal cavity of the fetus; midline celiotomy with the exposure of the pregnant uterus followed by injection through the uterine wall into the peritoneal cavity of the lamb or direct ultrasound-guided injections into the intracelomic cavity of the fetus $(11,12,13)$.

The aim of this study was to asses the feasibility of in utero human stem cell transplantation using an animal model and to compare two different techniques of performing in utero transplantation.

\section{Materials and Methods}

\section{A. Human Cord Blood Stem Cell Collection and Isolation}

Human cord blood from term deliveries were obtained after written informed consent in "Dominic Stanca” Clinic of Obstetrics and Gynecology, Cluj Napoca.

Mononuclear cells were isolated from the cord blood by Ficoll-Hypaque method, washed, and resuspended in PBS (Sigma). Before transplantation, the CD34+ human cord blood stem cell graft was washed in phosphate-buffered saline solution (PBS from Sigma), and resuspended at a concentration of $1 \times 10^{6}$ per $1 \mathrm{ml}$ IMDM (Sigma).

\section{B. Fetal Sheep Recipients}

10 Merinos sheep were selected for this study, divided into 3 groups. First group containing 4 sheep was subject to in utero stem cell transplantation using the midline celiotomy tecnique; the second group of 3 sheep was exposed to in utero transplantation by ultrasound-guided transabdominal percutaneous needle puncture technique and the last group was used as a control. Pregnancy and the gestational age of the fetuses was confirmed by ultrasound examination. 


\section{In utero stem cell transplantation}

Animals were placed in dorsal recumbancy, and the lower abdomen and mammary glands shaven and sterilized. For ultrasound and transplantation, the table was adjusted such that the head was lowered relative to the uterus at a 30-degree angle, which significantly reduced the difficulties in obtaining a clear view of the fetuses.

1) The ultrasound-guided transabdominal percutaneous needle puncture technique: Transabdominal ultrasound with a $3.5 \mathrm{MHz}$ curved array probe (Hitachi) was used to determine the position and viability of the fetuses, and to measure crown-rump length to confirm the gestational age (figure 1).

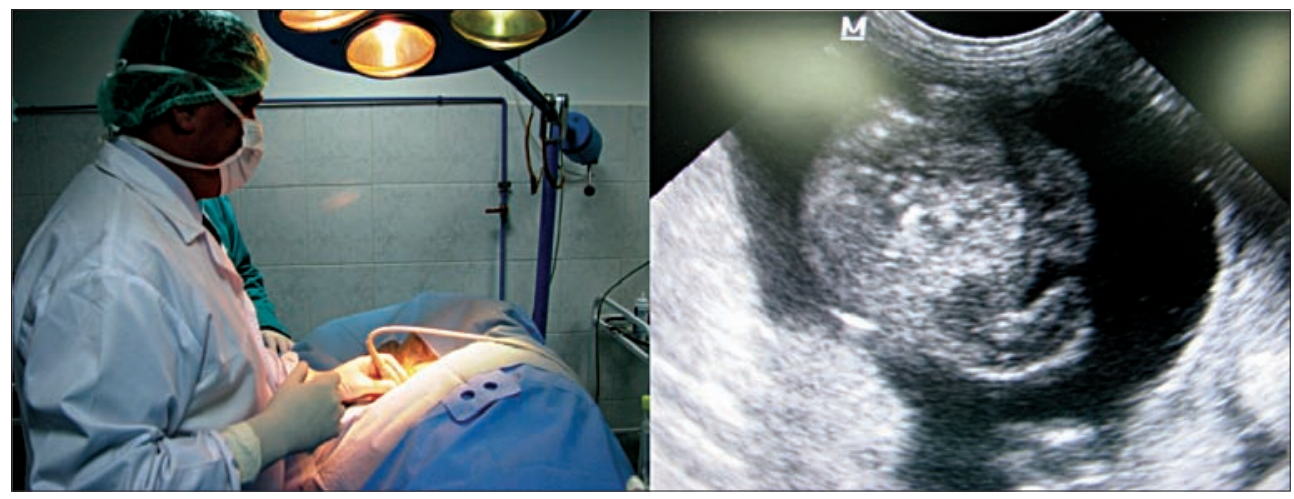

Fig. 1. Transabdominal ultrasound before in utero transplantation

A 20-gauge spinal needle was inserted through the skin and the uterine wall into the amniotic cavity and then into the peritoneal cavity of the fetuses under continuous ultrasound guidance by using the freehand technique. Care was taken not to insert the needle into the fetal liver. After confirmation of the appropriate positioning of the needle, the graft containing human stem cells was slowly injected in a total volume of $1 \mathrm{ml}$ (figure 2).

During infusion, the distribution of the fluid in the peritoneal cavity could be observed. The fetus was then checked to ensure adequate heartbeat after transplantation, and anesthetic was withdrawn from the ewe.

b) The midline celiotomy technique: After pre-surgical care and ultrasound examination, as described above, a midline celiotomy incision was performed using a blade and the pregnant uterus was delivered onto the operating field (figure 3).

The fetal position was assesed by palpation and a 20gauge needle was inserted trough the uterine wall into the peritoneal cavity of the fetus. A content of $1 \mathrm{ml}$ stem cell graft was slowly injected followed by the retraction of the needle. Exposed uteruses were warmed with periodic lavages with warmed normal saline. Same as before, the fetus was then checked to ensure adequate heartbeat after transplantation, and anesthetic was withdrawn from the ewe.

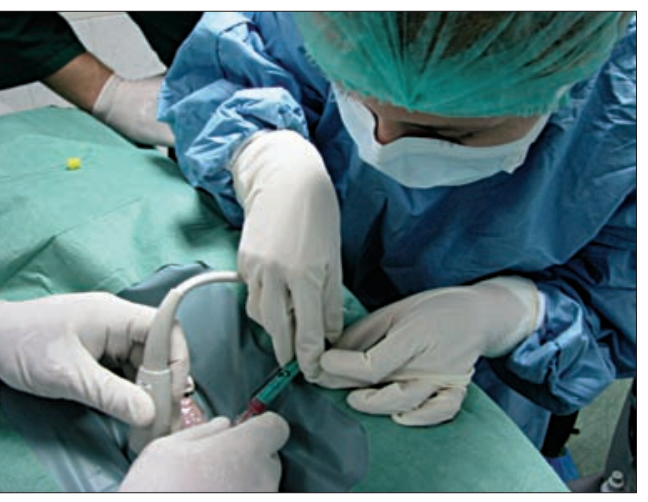

Fig. 2. In utero transplantation using the ultrasound-guided transabdominal percutaneous needle puncture technique

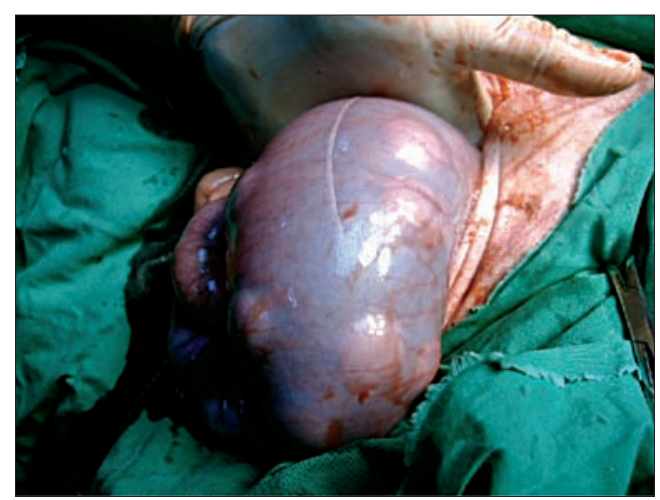

Fig. 3. In utero transplantation using the midline celiotomy technique 
Ewes were monitored for 2 weeks after the procedure for behavior, health, food intake, and signs of spontaneous abortion.

\section{Analysis of Human Stem Cell Engraftment}

10 to $20 \mathrm{~mL}$ of peripheral blood and bone marrow aspirates were harvested from the previously transplanted lambs, and prepared according to standard procedures. Cells were stained with an antihuman antibody specific for the human leukocyte common antigen, CD45 (BD Biosciences, San Jose, Ca) and with an antibody specific for hematopoietic stem cells CD 34 (BD Biosciences, San Jose, Ca).

Data were analyzed on a BD FACS Canto II (BD Biosciences, San Jose, Ca).

\section{Results}

In utero stem cell transplantation was performed in 7 ewes carrying a total of 7 fetuses ( 4 ewes from the first group and 3 ewes from the second group). The in utero stem cell transplantation was successfully at a $100 \%$ rate.

Three lambs were born at term, corresponding to a fetal loss rate of 42,85\% (3/7). The lost fetuses were either resorbed or aborted, mostly between 7 to 14 days after in utero transplantation. In the first group we had 2 abortions and 1 death, corresponding to a fetal loss rate of 75\% (3/4) (ewes in this group were transplanted using the the midline celiotomy technique). The second group of ewes were transplanted using the ultrasound-guided transabdominal percutaneous needle puncture technique and here we had a fetal loss rate of $33.3 \%(1 / 3)$

A total number of 3 lambs were available for engraftment analysis. Engraftment levels were between $1.3 \%$ and $1.4 \%$ of peripheral blood cells and between $3.3 \%$ and $3.7 \%$ of bone marrow cells (figure 4).
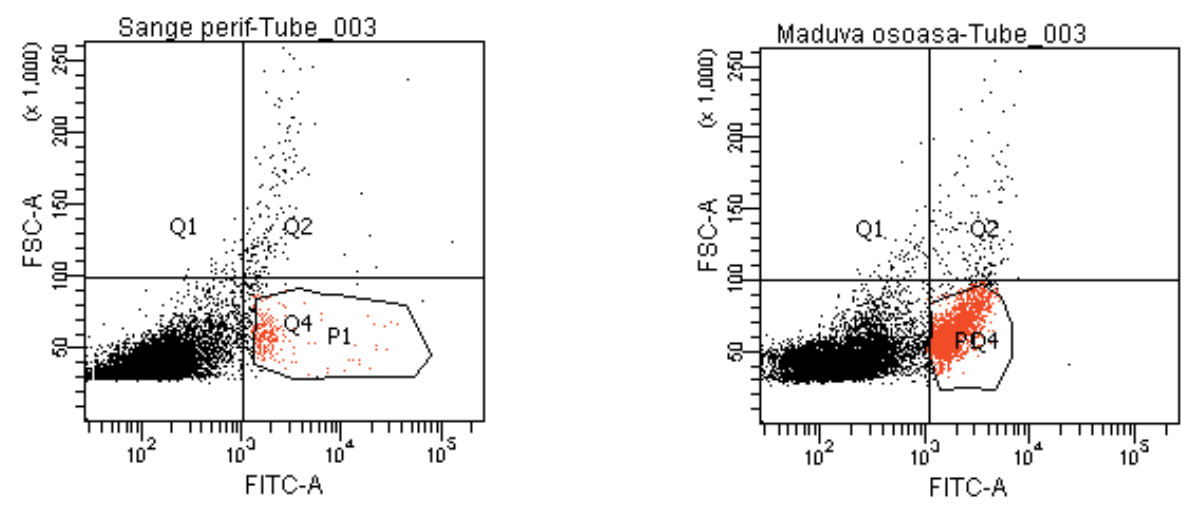

Fig. 4. The results of the FACS analysis for peripheral blood and bone marrow

\section{Discussion}

We were able to show for the first time in an Romanien university that in utero transplantation of human CD34+ stem cells leads to successful engraftment in fetal sheep peripheral blood and bone marrow. The level of engraftment was low in our study. It was, however, not our main purpose to achieve high-level engraftment. Some groups have reported higher engraftment levels using human cells in preimmune fetal sheep (14). The low engraftment levels may be due to the lack of human stromal elements supporting hematopoietic cells in homing, proliferation, and differentiation. Cotransplantation of human stromal elements has been shown to increase engraftment levels (15). 
We used two different techniques for in utero stem cell transplantation, both of them prouving to produce engraftment of human stem cells in fetal lamb recipients. Comparing the two techniques in terms of fetal loss, we concluded that the ultrasound-guided transabdominal percutaneous needle puncture technique is more effective than

the midline celiotomy technique.

This technique has a low fetal loss rate (33.3\%), marginally above the natural loss rate. Although this natural loss varies between breeds, it is estimated to be between $15 \%$ to $20 \%$. Our data prouved to be similar to the data already published (16).

The second technique used in our study had a fetal loss rate of $75 \%$; our data was above the one reported in literature to be as high as $50 \%$ (11).

The differences in fetal loss rate between the two techniques suggest that the surgical trauma associated with midline celiotomy and exposure of the pregnant uterus is more of a factor in fetal loss than specific effects that are due to the introduction of human cells in the sheep fetus.

\section{Conclusion}

In utero transplantation of hematopoietic stem cells has recently been shown to be an effective therapy for human fetuses affected by severe immunodeficiency (17) but not in nonimmunodeficient fetuses (eg, affected by hemoglobinopathy) (18). A valuable animal model is therefore crucial to develop improved strategies for clinical protocols aiming to improve engraftment levels to achieve clinical benefit. As described before, the fetal sheep model has several advantages over other animal models and has therefore become a valuable large animal model for prenatal stem cell transplantation $(5,6)$.

In our study we obtained the first sheep chimeras in Romania, performing in utero stem cell transplantation of human hematopoietic stem cells from umbilical cord blood.

We have demonstrated for the first time in Romania that in utero stem cell transplantation into sheep fetuses at an early gestational age is feasible and it is associated with engraftment in hematopoietic tissues, such as bone marrow and peripheral blood. The relevance of the ovine animal model to evaluate human stem cell activity must be careffuly addressed in further studies with longer animal follow-up and secondary stem cell transplants.

\section{References}

1. Muench MO. In utero transplantation: baby step towards an effective therapy. Bone Marrow Transplantation 2005; 35:537-47.

2. Flake AW, Zanjani ED. In utero hematopoietic stem cell transplantation: ontogenic opportunities and biologic barriers. Blood 1999; 94:2179-91.

3. Flake AW.,Zanjani ED. In utero hematopoietic stem cell transplantation. A status report. JAMA 1997, vol. 278, no. 11,932937.

4. Westgren M. In utero stem cell transplantation. Semin. Reprod. Med 2006; 24:348-357.

5. Almeida-Porada G, Porada C, Gupta N, Torabi A, Thain D, Zanjani ED. The human-sheep chimeras as a model for human stem cell mobilization and evaluation of hematopoietic graft's potential. Exp Hematol. 2007 Oct;35(10):1594-600

6. Porada GA, Porada C, Zanjani ED. The fetal sheep: a unique model system for assesing the full differentiative potential of human stem cells. Yonsei Med J. 2004 Jun 30; 45 Suppl: 7-14.

7. Porada CD, Park P, Almeida-Porada G, et al. The sheep model of in utero gene therapy. Fetal Diagn Ther 2004;19:23-30.

8. Miyasaka M, Morris B. The ontogeny of the lymphoid system and immune responsiveness in sheep. Prog Vet Micorbiol Immunol 1988; 4:21-55. 
9. Almeida-Porada G, Porada CD, Tran N, Zanjani ED. Cotransplantation of human stromal cell progenitors into preimmune fetal sheep results in early appearance of human donor cells in circulation and boosts cell levels in bone marrow at later time points after transplantation. Blood 2000;95:3620-7.

10. Surbek DV, Young A, Danzer E, et al. Ultrasound-guided stem cell sampling from the early ovine fetus for prenatal ex vivo gene therapy. Am J Obstet Gynecol 2002; 187:960-3.

11. Schoeberlein A, Holzgreve W, Dudler L, et al. In utero transplantation of autologous and allogeneic fetal liver stem cells in ovine fetuses. Am J Obstet Gynecol 2004;191:1030-6.

12. Bernstein J, Boyle DW, Srour EF, et al. Variation in long-term engraftment of a large consecutive series of lambs transplanted in utero with human hematopoietic cells. Biol Blood Marrow Transplant 1997; $3: 247-54$.

13. Dahm-Kähler P, Wranning C, Lundmark C, Enskog A, Mölne J, Marcickiewicz J, El-Akouri RR, McCracken J, Brännström M. Transplantation of the uterus in sheep: methodology and early reperfusion events. J Obstet Gynaecol Res. 2008 Oct;34(5):784-93.

14. Schoeberlein A, Holzgreve W, Dudler L, Hahn S, Surbek DV. Tissue-specific engraftment after in utero transplantation of allogenic mesenchymal stem cells into sheep fetuses. Am J Obstet Gynecol. 2005 Apr; 192(4):1044-52.

15. Airey JA, Almeida-Porada G, Colletti EJ, Porada CD, Chamberlain J, Movsesian M, Sutko JL, Zanjani ED. Human mesenchymal stem cells form Purkinje fibers in fetal sheep heart. Circulation. 2004 Mar 23; 109(11):1401-7. Epub 2004 Mar 15.

16. Oppenheim SM, Muench MO, Gutiérrez-Adán A, Moyer AL, BonDurant RH, Rowe JD, Anderson GB. Hematopoietic stem cell transplantation in utero produces sheep-goat chimeras. Blood Cells Mol Dis. 2001 Jan-Feb; 27(1):296-308.

17.Flake AW, Roncarolo MG, Puck JM, Almeida-Porada G, Evans MI,Johnson MP, et al. Treatment of Xlinked severe combined immunodeficiency by in utero transplantation of paternal bone marrow. $\mathrm{N}$ Engl J Med 1996; 335:1806-10.

18. Westgren M, Ringden O, Eik-Nes S, Ek S, Anvret M, Brubakk AM, et al. Lack of evidence of permanent engraftment after in utero fetal stem cell transplantation in congenital hemoglobinopathies. Transplantation 1996; 61:1176-9. 